\title{
POSITIVE TRIGONOMETRIC QUADRATURE FORMULAS AND QUADRATURE ON THE UNIT CIRCLE
}

\author{
FRANZ PEHERSTORFER
}

\begin{abstract}
We give several descriptions of positive quadrature formulas which are exact for trigonometric-, respectively, Laurent polynomials of degree less or equal to $n-1-m, 0 \leq m \leq n-1$. A complete and simple description is obtained with the help of orthogonal polynomials on the unit circle. In particular it is shown that the nodes polynomial can be generated by a simple recurrence relation. As a byproduct interlacing properties of zeros of paraorthogonal polynomials are obtained. Finally, asymptotics for the quadrature weights are presented.
\end{abstract}

\section{INTRODUCTION}

Let $l \in \mathbb{N}_{0}:=\mathbb{N} \cup\{0\}, \gamma \in\{0,1 / 2\}$, and let $\mathcal{T}_{l, \gamma}=\left\{\sum_{k=0}^{l} a_{k} \cos (k+\gamma) \varphi+\right.$ $\left.b_{k} \sin (k+\gamma) \varphi: a_{k}, b_{k} \in \mathbb{R}\right\}$. By $\mathcal{T}_{l}:=\mathcal{T}_{l, 0}$ we denote the set of trigonometric polynomials of degree less or equal to $l$. Further let $\sigma$ be a positive measure on $[0,2 \pi]$ normalized by $\frac{1}{2 \pi} \int_{0}^{2 \pi} d \sigma=1$. We call a quadrature formula (qf) of the form

$$
\frac{1}{2 \pi} \int_{0}^{2 \pi} t(\varphi) d \sigma(\varphi)=\sum_{s=1}^{n} \mu_{s} t\left(\varphi_{s}\right)+R_{n}(t)
$$

with $0 \leq \varphi_{1}<\varphi_{2}<\cdots<\varphi_{n}<2 \pi, \mu_{1}, \ldots, \mu_{n} \in \mathbb{R}$, a trigonometric $(n-1-m, n, d \sigma)$ qf if $R_{n}(t)=0$ for $t \in \mathcal{T}_{n-1-m} ; n-1-m$ is the so-called degree of exactness. Note that for $n \in \mathbb{N}, n=2(\tilde{n}+\gamma), \gamma \in\{0,1 / 2\}$, the trigonometric nodes polynomial

$$
\prod_{s=1}^{n} \sin \left(\frac{\varphi-\varphi_{s}}{2}\right)=e^{-i\left(n \varphi+\sum_{s=1}^{n} \varphi_{s}\right) / 2} \prod_{s=1}^{n}\left(e^{i \varphi}-e^{i \varphi_{s}}\right) / 2 i
$$

is from $\mathcal{T}_{\tilde{n}, \gamma}$.

Obviously, (1) is a $(n-1-m, n, d \sigma)$ qf if and only if

$$
\frac{1}{2 \pi} \int_{0}^{2 \pi} e^{-i k \varphi} d \sigma(\varphi)=\sum_{s=1}^{n} \mu_{s} e^{-i k \varphi_{s}} \quad k=0, \ldots, \pm(n-1-m),
$$

Received by the editor November 6, 2008 and, in revised form, January 29, 2010.

2010 Mathematics Subject Classification. Primary 65D30, 41A55, 42C05.

Key words and phrases. Trigonometric quadrature formulas, orthogonal polynomials, positive quadrature weights, recurrence relation, para-orthogonal polynomials, zeros, asymptotics.

The author was supported by the Austrian Science Fund FWF, project no. P20413-N18.

Sadly, on November 27, 2009 the author, Franz Peherstorfer, passed away. Since then, Ionela Moale, his last PhD student, did the main work in preparing the paper for publication, and Franz's colleague, Professor Peter Yuditskii, has kindly stepped in to proofread the galley proof of the work. 
which is a qf on the unit circle exactly for Laurent polynomials $\sum_{-(n-1-m)}^{n-1-m} d_{k} z^{k}$, $d_{k} \in \mathbb{C}$ of degree $\leq n-m-1$, which we call $(n-1-m, n, d \sigma)$ a Laurent qf also. In other words, we are looking for a characterization of quadrature formulas which integrate exactly the first $n-1-m$ moments $c_{1}, \ldots, c_{n-1-m}$ of the Caratheodory function

$$
F(z)=\frac{1}{2 \pi} \int_{0}^{2 \pi} \frac{e^{i \varphi}+z}{e^{i \varphi}-z} d \sigma(\varphi)=1+2 \sum_{k=1}^{\infty} c_{k} z^{k} .
$$

Due to convergence and stability it is of utmost interest to have positive ( $n-$ $1-m, n, d \sigma)$ qf, i.e., that all quadrature weights $\mu_{s}$ are positive. Such qf will be studied in this paper. So far it is known that qf with the highest possible degree of exactness, that is, $(n-1, n, d \sigma)$ qf have positive weights. These $(n-1, n, d \sigma)$ qf can be considered as the counterparts of the Gauss qf for algebraic polynomials and nowadays are called Szegö qf. The Szegö qf can be described as follows (see [8. pp. 14-16], in particular (10.10), but also [1, 13, 22, 26]): $\varphi_{1}, \ldots, \varphi_{n}, 0 \leq$ $\varphi_{1}<\cdots<\varphi_{n}<2 \pi$, generate a $(n-1, n, d \sigma)$ Laurent qf if and only if there is $\eta \in \mathbb{T}:=\{z \in \mathbb{C}:|z|=1\}$ such that

$$
z \Phi_{n-1}(z)+\eta \Phi_{n-1}^{*}(z)=\prod_{s=1}^{n}\left(z-e^{i \varphi_{s}}\right)
$$

Here and in what follows $\Phi_{n}(z)=z^{n}+\ldots$ always denotes the monic polynomial of degree $n$ orthogonal with respect to the normalized measure $d \sigma$, i.e.,

$$
\int_{0}^{2 \pi} e^{-i k \varphi} \Phi_{n}\left(e^{i \varphi}\right) d \sigma(\varphi)=0 \quad k=0, \ldots, n-1 .
$$

It is well known that the $\Phi_{n}$ 's satisfy a recurrence relation of the form

$$
\Phi_{n}(z)=z \Phi_{n-1}(z)-a_{n-1} \Phi_{n-1}^{*}(z),
$$

with $\left|a_{j}\right|<1$ for $j=0,1,2, \ldots$ and where $\Phi_{n-1}^{*}(z)=z^{n-1} \bar{\Phi}_{n-1}\left(\frac{1}{z}\right)$; hence

$$
\Phi_{n}^{*}(z)=\Phi_{n-1}^{*}(z)-\bar{a}_{n-1} z \Phi_{n-1}(z) .
$$

Furthermore, let us recall that for any $\kappa \in \mathbb{T}$,

$$
z \Phi_{n-1}+\kappa \Phi_{n-1}^{*}=\prod_{s=1}^{n}\left(z-e^{i \psi_{s}}\right)
$$

where $0 \leq \psi_{1}<\cdots<\psi_{n}<2 \pi$, since $\Phi_{n-1}$ has all zeros in $\mathbb{D}=\{|z|<1\}$. Following [13] a polynomial of the form (8) is called a para-orthogonal polynomial.

The Szegö quadrature weights are given by, $z_{s}=e^{i \psi_{s}}$,

$$
\mu_{s}=\frac{1}{2 z_{s}} \frac{\left(z \Psi_{n-1}-\eta \Psi_{n-1}^{*}\right)\left(z_{s}\right)}{\left(z \Phi_{n-1}+\eta \Phi_{n-1}^{*}\right)^{\prime}\left(z_{s}\right)}>0
$$

where $\Psi_{n}$ is the so-called polynomial of the second kind, i.e.,

$$
\Psi_{n}(z)=\frac{1}{2 \pi} \int_{0}^{2 \pi} \frac{z+e^{i \varphi}}{z-e^{i \varphi}}\left(\Phi_{n}(z)-\Phi_{n}\left(e^{i \varphi}\right)\right) d \sigma(\varphi)
$$

which satisfies the recurrence relation

$$
\Psi_{n}(z)=z \Psi_{n-1}(z)+a_{n-1} \Psi_{n-1}^{*}(z) .
$$


Note that $a_{n-1}$ in (6) is just replaced by $-a_{n-1}$. Recently, Szegó quadrature formulas have been studied and applied by several authors [4, 6, 11, 10, 14. For para-orthogonal polynomials see [5, 24, 27].

As mentioned already, here we study positive $(n-1-m, n, d \sigma)$ qf. There are two possible approaches, one approach is comparable to the algebraic polynomial approach and the other one via orthogonal polynomials on the unit circle (abbreviated by OPUC). Each of which has its advantages as we shall see. In the next section we derive some characterizations without using OPUC's. In fact, for Theorem 2.2. we do not even know how to obtain it via OPUC's and for the other properties as the orthogonality condition given in Lemma 2.1, they follow in a more natural way than with the help of OPUC's; compare Lemma 3.5 and Corollary 3.6. In Section 3 we give a simple complete description of positive trigonometric-, resp., Laurent $\mathrm{qf}$ with the help of OPUC's. In this problem the power of the approach via the unit circle turns out nicely. We note that Section 3 and Section 4 can be read without having studied Section 2.

\section{BASIC CHARACTERIZATION}

First let us take a look at the degree of exactness. Similarly, as in the case of algebraic polynomials, the degree of exactness is connected with the orthogonality property of the nodes polynomial. For Szegó qf see [3] and for the associated orthogonal trigonometric polynomials and their five-term recurrence relation see 12 .

Lemma 2.1. Let $n \in \mathbb{N}, n=2(\tilde{n}+\gamma), \gamma \in\{0,1 / 2\}$. A qf of the form (1) is exact for $t \in \mathcal{T}_{\tilde{n}+2 \gamma+l}, l \in \mathbb{N}_{0}$, if and only if the trigonometric nodes polynomial $T_{\tilde{n}, \gamma}(\varphi)$ satisfies the orthogonality conditions

$$
\int_{0}^{2 \pi} t(\varphi) T_{\tilde{n}, \gamma}(\varphi) d \sigma(\varphi)=0 \text { for } t \in \mathcal{T}_{l, \gamma}
$$

Proof. Case 1) $n=2 \tilde{n}$, i.e., $\gamma=0$.

Sufficiency. First let us consider the case $l=0$. Let $\xi \in[0,2 \pi)$ be no zero of $T_{\tilde{n}}$ and arbitrary otherwise. Since the Lagrange trigonometric polynomials with respect to the nodes, $\varphi_{1}, \ldots, \varphi_{2 \tilde{n}}$ and $\xi$ are polynomials from $\mathcal{T}_{\tilde{n}}$, the interpolation quadrature formula

$$
\int_{0}^{2 \pi} t(\varphi) d \sigma=\sum_{s=1}^{2 \tilde{n}} \mu_{s} t\left(\varphi_{s}\right)+\mu_{2 \tilde{n}+1} t(\xi)
$$

holds for every $t \in \mathcal{T}_{\tilde{n}}$ and is unique. Now any $q \in \mathcal{T}_{\tilde{n}}$ can be represented in the form

$$
q(\varphi)=c T_{\tilde{n}}(\varphi)+t(\varphi)
$$

where $t \in \mathcal{T}_{\tilde{n}}$ is the unique polynomial satisfying the interpolation conditions $t(\xi)=$ 0 and $t\left(\varphi_{s}\right)=q\left(\varphi_{s}\right)$ for $s=1, \ldots, 2 \tilde{n}$ and $c=q(\xi) / T_{\tilde{n}}(\xi)$. Hence, applying (13) to $q$ and taking into consideration the orthogonality property of $T_{\tilde{n}}$, we obtain

$$
\frac{1}{2 \pi} \int_{0}^{2 \pi} q d \sigma=\frac{1}{2 \pi} \int_{0}^{2 \pi} c T_{\tilde{n}} d \sigma+\frac{1}{2 \pi} \int_{0}^{2 \pi} t d \sigma=\sum_{s=1}^{2 \tilde{n}} \mu_{s} t\left(\varphi_{s}\right)=\sum_{s=1}^{2 \tilde{n}} \mu_{s} q\left(\varphi_{s}\right) .
$$


In the case of a higher orthogonality property of $T_{\tilde{n}}$ we represent $q \in \mathcal{T}_{\tilde{n}+l}$ in the form

$$
q=v T_{\tilde{n}}+t
$$

where $t$ is as above and $v \in \mathcal{T}_{l}$ and the assertion follow.

The necessity part follows immediately by applying the quadrature formula to $t T_{\tilde{n}}$.

Case 2) $n=2 \tilde{n}+1$, i.e., $\gamma=1 / 2$.

Sufficiency. Any $q \in \mathcal{T}_{\tilde{n}+1+l}$ can be represented in the form

$$
q=v_{l, 1 / 2} T_{\tilde{n}, 1 / 2}+t
$$

where $t \in \mathcal{T}_{\tilde{n}}$ interpolates $q$ at the $2 \tilde{n}+1$ zeros of $T_{\tilde{n}, 1 / 2}$ and where $v_{l, 1 / 2} \in \mathcal{T}_{l, 1 / 2}$. Applying the unique $(\tilde{n}, 2 \tilde{n}+1, d \sigma)$ quadrature formula (13) to $q$ and writing $\xi=$ $\varphi_{2 \tilde{n}+1}$, the assertion follows by the orthogonality property.

Necessity is immediate again.

We note that if $l<\tilde{n}-1$, then the orthogonality property (12) does not imply that all zeros of $T_{\tilde{n}, \gamma}$ are simple and in $[0,2 \pi)$ there may also appear double or complex zeros.

To find a criterion on the nodes polynomial such that the quadrature weights are positive is more involved (in contrast to the algebraic polynomial case, where the corresponding counterpart can be derived in one line; see e.g. [20, (1.3)].

Theorem 2.2. Let $n \in \mathbb{N} \backslash\{1\}, n=2(\tilde{n}+\gamma), \gamma \in\{0,1 / 2\}$, and let (1) be a $(\tilde{n}+2 \gamma, 2(\tilde{n}+\gamma), d \sigma)$ qf. Denote by $T(\varphi)$ the trigonometric nodes polynomial, which is from $\mathcal{T}_{\tilde{n}, \gamma}$, and write

$$
S(\psi)=\frac{1}{2 \pi} \int_{0}^{2 \pi} \cot \left(\frac{\varphi-\psi}{2}\right)(T(\psi)-T(\varphi)) d \sigma(\varphi) .
$$

Then $S(\psi) \in \mathcal{T}_{\tilde{n}, \gamma}$ and

$$
\mu_{s}=-\frac{S\left(\varphi_{s}\right)}{2 T^{\prime}\left(\varphi_{s}\right)} \text { for } s=1, \ldots, n .
$$

In particular, all quadrature weights $\mu_{s}$ are positive if and only if $S(\varphi)$ has $n$ simple zeros $\theta_{j}, 0 \leq \theta_{1}<\theta_{2}<\cdots<\theta_{n}<2 \pi$, which separate the zeros $\varphi_{j}$ of $T(\varphi)$ such that $\varphi_{1}<\theta_{1}<\varphi_{2}<\cdots$ if $\operatorname{sgn}(S T)\left(0^{+}\right)>0$, respectively, $\theta_{1}<\varphi_{1}<\theta_{2}<\cdots$ if $\operatorname{sgn}(S T)\left(0^{+}\right)<0$.

Furthermore, setting

$$
\tau_{n}\left(e^{i \varphi}\right)=e^{i \frac{n}{2} \varphi} T(\varphi) \text { and } \omega_{n}\left(e^{i \varphi}\right)=i e^{i \frac{n}{2} \varphi} S(\varphi),
$$

the ratio of the polynomials $\omega_{n}(z)$ and $\tau_{n}(z)$ has an expansion of the form

$$
-\frac{\omega_{n}(z)}{\tau_{n}(z)}=\sum_{s=1}^{n} \mu_{s} \frac{e^{i \varphi_{s}}+z}{e^{i \varphi_{s}}-z}=1+2 \sum_{k=1}^{\tilde{n}+2 \gamma+l} c_{k} z^{k}+\ldots
$$

where $c_{k}=\int_{0}^{2 \pi} e^{-i k \varphi} d \sigma(\varphi)$ and $\tilde{n}+2 \gamma+l$ is the precise degree of exactness of the qf (11).

Proof. Let $R(\varphi) \in \mathcal{T}_{\tilde{n}, \gamma}$ be the polynomial such that, $z=e^{i \varphi}$,

$$
\frac{i e^{i \frac{n}{2} \varphi} R(\varphi)}{e^{i \frac{n}{2} \varphi} T(\varphi)}=\sum_{s=1}^{n} \mu_{s} \frac{z+e^{i \varphi_{s}}}{z-e^{i \varphi_{s}}}
$$


Putting $\omega_{n}\left(e^{i \varphi}\right)=i e^{i \frac{n}{2} \varphi} R(\varphi)$ and $\tau_{n}\left(e^{i \varphi}\right)=e^{i \frac{n}{2} \varphi} T(\varphi)$ we may rewrite equation (18) in the form

$$
\frac{\omega_{n}(z)}{\tau_{n}(z)}-1=-2 \sum_{s=1}^{n} \frac{\mu_{s}}{1-e^{-i \varphi_{s} z}}
$$

where we have used the fact that

$$
\sum_{s=1}^{n} \mu_{s}=\frac{1}{2 \pi} \int d \sigma=1
$$

since the qf is exact for constants and $\sigma$ is normalized. Note that $\omega_{n}(0)=-\tau_{n}(0)$ or, in other words, that

(20) $T(\varphi)=a_{n} \cos \frac{n}{2} \varphi+b_{n} \sin \frac{n}{2} \varphi+\ldots$ and $R(\varphi)=-b_{n} \cos \frac{n}{2} \varphi+a_{n} \sin \frac{n}{2} \varphi+\ldots$

Furthermore, it follows from (19) that

$$
\frac{\omega_{n}\left(e^{i \varphi_{s}}\right)}{\frac{d \tau_{n}}{d z}\left(e^{i \varphi_{s}}\right)}=2 \mu_{s} e^{i \varphi_{s}}
$$

which yields that

$$
\frac{i R\left(\varphi_{s}\right)}{\frac{d T}{d \varphi}\left(\varphi_{s}\right)}=\frac{e^{-i \frac{n}{2} \varphi_{s}} \omega_{n}\left(e^{i \varphi_{s}}\right)}{\frac{d}{d \varphi}\left(e^{-i \frac{n}{2} \varphi_{s}} \tau_{n}\left(e^{i \varphi}\right)\right)_{\varphi=\varphi_{s}}}=\frac{\omega_{n}\left(e^{i \varphi_{s}}\right)}{\frac{d}{d \varphi}\left(\tau_{n}\left(e^{i \varphi}\right)\right)_{\varphi=\varphi_{s}}}=\frac{2 \mu_{s}}{i} .
$$

The goal is to show that

$$
S \equiv R
$$

Let us prove (22) when $n$ is even.

Using the facts that

$$
\cot \left(\frac{\varphi-\psi}{2}\right)(\cos \psi-\cos \varphi)=\sin \psi+\sin \varphi
$$

and that

$$
\frac{\cos k \psi-\cos k \varphi}{\cos \psi-\cos \varphi}=2 \cos (k-1) \psi+\ldots
$$

is a cosine polynomial of degree $k-1$ in $\psi$ with leading coefficient 2 , it follows by straightforward calculation that for $k \in \mathbb{N}$,

$$
\begin{aligned}
& \int_{0}^{2 \pi} \cot \left(\frac{\varphi-\psi}{2}\right)(\cos k \psi-\cos k \varphi) d \sigma(\varphi) \\
& =\int_{0}^{2 \pi} \cot \left(\frac{\varphi-\psi}{2}\right)(\cos \psi-\cos \varphi)\left(\frac{\cos k \psi-\cos k \varphi}{\cos \psi-\cos \varphi}\right) d \sigma(\varphi) \\
& =\left(\int_{0}^{2 \pi} d \sigma\right) \sin k \psi+t(\psi)
\end{aligned}
$$


where $t \in \mathcal{T}_{k-1}$. Furthermore,

$$
\begin{aligned}
& \int_{0}^{2 \pi} \cot \left(\frac{\varphi-\psi}{2}\right)(\sin k \psi-\sin k \varphi) d \sigma(\varphi) \\
& =\sin \psi \int_{0}^{2 \pi} \cot \left(\frac{\varphi-\psi}{2}\right)\left(\frac{\sin k \psi}{\sin \psi}-\frac{\sin k \varphi}{\sin \varphi}\right) d \sigma(\varphi) \\
& +\int_{0}^{2 \pi} \cot \left(\frac{\varphi-\psi}{2}\right)(\sin \psi-\sin \varphi) \frac{\sin k \varphi}{\sin \varphi} d \sigma(\varphi) \\
& =-\left(\int_{0}^{2 \pi} d \sigma\right) \cos k \psi+t(\psi)
\end{aligned}
$$

where $t \in \mathcal{T}_{k-1}$, and where in the last equality we used the facts that $\sin k x / \sin x=$ $2 \cos (k-1) x+\ldots$ is a cosine polynomial of degree $k-1$ in $x$ and thus (24) can be applied to the first integral and that the second integral is from $\mathcal{T}_{1}$. Thus $S(\psi) \in \mathcal{T}_{\tilde{n}}$ and by (20) it follows that $S$ and $R$ have the same leading coefficients, i.e., $R-S \in \mathcal{T}_{\tilde{n}-1}$. Writing $T(\varphi)$ in terms of $\sin \left(\frac{\varphi-\varphi_{s}}{2}\right)$ it follows that the integrand from (15) at $\psi=\varphi_{s}$ is from $\mathcal{T}_{\tilde{n}}$ and thus we may apply the quadrature formula to the integrand and obtain

$$
S\left(\varphi_{s}\right)=-\mu_{s} 2 T^{\prime}\left(\varphi_{s}\right)=R\left(\varphi_{s}\right), s=1, \ldots, 2 \tilde{n},
$$

where the last equality follows by (21). Hence, $S \equiv R$ and the assertion is proved for $n$ even.

For odd $n$ we consider

$$
\tilde{S}(\psi)=\frac{1}{2 \pi} \int_{0}^{2 \pi} \cot \left(\frac{\varphi-\psi}{2}\right)((q T)(\psi)-(q T)(\varphi)) d \sigma(\varphi)
$$

where $q$ is any polynomial from $\mathcal{T}_{0,1 / 2}$ with $q(\varphi)>0$ on $[0,2 \pi)$. Since $q T \in \mathcal{T}_{\tilde{n}+1}$ it follows by (24) and (25) that $\tilde{S} \in \mathcal{T}_{\tilde{n}+1}$. Furthermore, we may apply the quadrature formula and obtain that

$$
\tilde{S}\left(\varphi_{s}\right)=-\mu_{s} 2 q\left(\varphi_{s}\right) T^{\prime}\left(\varphi_{s}\right)=q\left(\varphi_{s}\right) R\left(\varphi_{s}\right) \text { for } s=1, \ldots, 2 \tilde{n}+1 .
$$

Next we claim that

$$
\tilde{S}(\psi)=q(\psi) S(\psi) .
$$

To prove (28) it suffices to show that

$$
\int_{0}^{2 \pi} \cot \left(\frac{\varphi-\psi}{2}\right)(q(\psi)-q(\varphi)) T(\varphi) d \sigma(\varphi)=0
$$

which follows immediately by $q(\psi)-q(\varphi)=$ const $\sin \left(\frac{\varphi-\psi}{2}\right)$ and the orthogonality property of $T$ with respect to $\mathcal{T}_{0,1 / 2}$.

Therefore, by (28) and (27), if we are able to show that the leading coefficients of $\tilde{S}(\varphi)$ and $(q R)(\varphi)$ coincide, i.e., $\tilde{S}-q R \in \mathcal{T}_{\tilde{n}}$, representation (15) follows for $n$ is odd also.

Because of (20) we know the leading coefficients of $(q T)(\varphi)$ and $(q R)(\varphi)$. By applying (24) and (25) to $(q T)(\psi)-(q T)(\varphi)$ it follows that the leading coefficients of $\tilde{S}$ are equal to those of $q R$. Hence, $\tilde{S}=q R$ and the theorem is proved.

The statement on the positivity of the $\mu_{s}$ follows immediately by (26).

The last equality in (17) follows by partial fraction expansion and (2). 
Theorem 2.2 (which turns out to be crucial in the Szegö-Kronrod quadrature 21. for instance) does not show how to obtain a constructive description of positive trigonometric qf. Such a description will be obtained by OPUC's in the next section.

\section{THE CHARACTERIZATION THEOREM}

First let us recall that the monic polynomials $\Phi_{n}(z)$ of degree $n, n \in \mathbb{N}_{0}$, are the polynomials orthogonal with respect to $d \sigma$, where $\frac{1}{2 \pi} \int_{0}^{2 \pi} d \sigma=1$, and that the $a_{j}$ 's are their recurrence coefficients; see (6).

Theorem 3.1. The following four statements are equivalent:

a)

$$
\frac{1}{2 \pi} \int_{0}^{2 \pi} e^{-i k \varphi} d \sigma(\varphi)=\sum_{s=1}^{n} \mu_{s} e^{-i k \varphi_{s}} \quad \text { for } k=0, \ldots, \pm(n-m-1)
$$

where $0 \leq \varphi_{1}<\varphi_{2}<\cdots<\varphi_{n}<2 \pi$ and $\mu_{1}, \ldots, \mu_{n} \in \mathbb{R}^{+}$.

b) There exists a polynomial $\tilde{\Phi}_{n-1}(z)=z^{n-1}+\ldots$ generated by the recurrence relation

$$
\tilde{\Phi}_{j}(z)=z \tilde{\Phi}_{j-1}(z)-\tilde{a}_{j-1} \tilde{\Phi}_{j-1}^{*}(z)
$$

where

$$
\left|\tilde{a}_{j}\right|<1 \text { for } j=0, \ldots, n-2 \text { and } \tilde{a}_{j}=a_{j} \text { for } j=0, \ldots, n-m-2 \text {, }
$$

and $a \eta \in \mathbb{T}$ such that

$$
z \tilde{\Phi}_{n-1}+\eta \tilde{\Phi}_{n-1}^{*}=\prod_{s=1}^{n}\left(z-e^{i \varphi_{s}}\right) .
$$

c) There exists a polynomial $q_{m}(z)=z^{m}+\ldots$ which has all its zeros in $\mathbb{D}$ and $\eta \in \mathbb{T}$ such that

$$
z q_{m} \Phi_{n-m-1}+\eta q_{m}^{*} \Phi_{n-m-1}^{*}=\prod_{s=1}^{n}\left(z-e^{i \varphi_{s}}\right) .
$$

d) There exists a polynomial $p_{n-1}(z)=z^{n-1}+\ldots$ which has all its zeros in $\mathbb{D}$ and $\eta \in \mathbb{T}$ such that

$$
z p_{n-1}+\eta p_{n-1}^{*}=\prod_{s=1}^{n}\left(z-e^{i \varphi_{s}}\right)
$$

and

$$
\frac{\eta p_{n-1}^{*}-z p_{n-1}}{\eta p_{n-1}^{*}+z p_{n-1}}=1+2 \sum_{k=1}^{n-m-1} c_{k} z^{k}+O\left(z^{n-m}\right)
$$

where $c_{k}=\frac{1}{2 \pi} \int_{0}^{2 \pi} e^{-i k \varphi} d \sigma(\varphi)$.

Proof. a) $\Rightarrow$ b) Since $\mu_{1}, \ldots, \mu_{n}>0$ it follows that the sequence

$$
\tilde{c}_{k}=\sum_{s=1}^{n} \mu_{s} e^{-i k \varphi_{s}} \quad k=0, \ldots, n-1
$$


is positive definite; see [1. Thus the polynomials $\tilde{\Phi}_{j}, j=0, \ldots, n-1$, orthogonal with respect to the positive definite sequence $\tilde{c}_{0}, \ldots, \tilde{c}_{n-1}$ satisfy a recurrence relation of the form (30) with $\left|\tilde{a}_{j}\right|<1$ for $j=0, \ldots, n-2$. Since by assumption

$$
\tilde{c}_{k}=\frac{1}{2 \pi} \int e^{-i k \varphi} d \sigma(\varphi) \quad k=0, \ldots, n-m-1
$$

it follows that (see e.g. [8, (3.2)])

$$
\tilde{a}_{k}=a_{k} \quad \text { for } k=0, \ldots, n-m-2 .
$$

Finally, we know [8, Thm. 6.1] that for any $a_{n-1}$ with $\left|a_{n-1}\right|=1$,

$$
\begin{array}{r}
-\frac{z \tilde{\Psi}_{n-1}-a_{n-1} \tilde{\Psi}_{n-1}^{*}}{z \tilde{\Phi}_{n-1}+a_{n-1} \tilde{\Phi}_{n-1}^{*}}=1+2 \sum_{k=1}^{n-1} \tilde{c}_{k} z^{k}+O\left(z^{n}\right) \\
=-\sum_{s=1}^{n} \mu_{s} \frac{z+e^{i \varphi_{s}}}{z-e^{i \varphi_{s}}}+O\left(z^{n}\right) .
\end{array}
$$

Putting $a_{n-1}=-e^{i \varphi_{1}} \tilde{\Phi}_{n-1}\left(e^{i \varphi_{1}}\right) / \tilde{\Phi}_{n-1}^{*}\left(e^{i \varphi_{1}}\right)$, that is, the denominator in (39) vanishes at $e^{i \varphi_{1}}$, it follows that (see $[8,(9.5)]$ )

$$
z \tilde{\Phi}_{n-1}+a_{n-1} \tilde{\Phi}_{n-1}^{*}=\prod_{s=1}^{n}\left(z-e^{i \varphi_{s}}\right) .
$$

b) $\Rightarrow$ a) By (30) and the first condition of (31), $\tilde{\Phi}_{n-1}(z)$, is orthogonal with respect to some positive measure $\tilde{\sigma}$. Thus (32) holds for any $\eta$ with $|\eta|=1$ and

$$
\frac{1}{2 \pi} \int_{-\pi}^{+\pi} e^{-i k \varphi} d \tilde{\sigma}(\varphi)=\sum_{s=1}^{n} \mu_{s} e^{-i k \varphi_{s}} \quad \text { for } k=0, \ldots, n-1
$$

where $\mu_{1}, \ldots, \mu_{n} \in \mathbb{R}^{+}$. Since $\tilde{a}_{j}=a_{j}$ for $j=0, \ldots, n-m-2$ it follows that $\tilde{\Phi}_{j}=\Phi_{j}$ and $\tilde{\Psi}_{j}=\Psi_{j}$ for $j=0, \ldots, n-m-1$ and thus the first $n-m-1$ moments of $\sigma$ and $\tilde{\sigma}$ coincide, that is,

$$
\int e^{-i k \varphi} d \tilde{\sigma}(\varphi)=\int e^{-i k \varphi} d \sigma(\varphi) \quad k=0, \ldots, n-m-1
$$

which gives by (41) the assertion.

b) $\Rightarrow$ c) Let

$$
q_{j}(z)=z q_{j-1}(z)-\eta \overline{\tilde{a}}_{n-1-j} q_{j-1}^{*}(z) \quad j=1,2, \ldots, m
$$

with $q_{0}(z)=1$. Then it follows by induction arguments using (6) and (7) that

$$
z \tilde{\Phi}_{n-1}+\eta \tilde{\Phi}_{n-1}^{*}=z q_{j} \tilde{\Phi}_{n-j}+\eta q_{j}^{*} \tilde{\Phi}_{n-j}^{*} .
$$

In view of (43) $q_{j}$ has all zeros in $\mathbb{D}$; see e.g. 22 .

We note that it follows analogously by induction arguments that

$$
z \tilde{\Psi}_{n-1}-\eta \tilde{\Psi}_{n-1}^{*}=z q_{j} \Psi_{n-j}-\eta q_{j}^{*} \Psi_{n-j}^{*} .
$$

c) $\Rightarrow$ b) Since $q_{m}(z)$ has all zeros in $\mathbb{D}, q_{m}$ can be generated by a recurrence relation of the form

$$
q_{j}(z)=z q_{j-1}(z)-\eta \bar{b}_{j-1} q_{j-1}(z) \quad j=1, \ldots, m
$$


where $\left|b_{j}\right|<1$ for $j=0, \ldots, m-1$ and $q_{0}(z)=1$. Hence, defining the polynomial $\tilde{\Phi}_{n-1}$ by the recurrence relation (6) with recurrence coefficients for OPUC's $a_{0}, \ldots, a_{n-m-1}, b_{m-1}, \ldots, b_{0}$ it follows as above that (32) holds, which implies b).

c) $\Rightarrow$ d) We put

$$
\begin{aligned}
& z q_{m} \Phi_{n-m-1}+\eta q_{m}^{*} \Phi_{n-m-1}^{*}=z p_{n-1}+\eta p_{n-1}^{*}, \\
& z q_{m} \Psi_{n-m-1}-\eta q_{m}^{*} \Psi_{n-m-1}^{*}=z p_{n-1}-\eta p_{n-1}^{*}
\end{aligned}
$$

that is,

$$
\begin{aligned}
& 2 z p_{n-1}=z q_{m}\left(\Phi_{n-m-1}+\Psi_{n-m-1}\right)+\eta q_{m}^{*}\left(\Phi_{n-m-1}^{*}-\Psi_{n-m-1}^{*}\right), \\
& 2 \eta p_{n-1}^{*}=z q_{m}\left(\Phi_{n-m-1}-\Psi_{n-m-1}\right)+\eta q_{m}^{*}\left(\Phi_{n-m-1}^{*}+\Psi_{n-m-1}^{*}\right) .
\end{aligned}
$$

Let us demonstrate that $p_{n-1}^{*}$ has no zero in $\overline{\mathbb{D}}$. It is known (see (1.3.82), (3.2.62) and (3.2.63)) from $\left[22\right.$ that $\Phi_{n-m-1}^{*}+\Psi_{n-m-1}^{*}$ has no zeros in $\overline{\mathbb{D}}$. We claim that

$$
\left|\frac{z\left(\Phi_{n-m-1}-\Psi_{n-m-1}\right)}{\Phi_{n-m-1}^{*}+\Psi_{n-m-1}^{*}}\right|<1 \quad \text { for }|z| \leq 1 .
$$

By the maximum principle it suffices to show (49) for $|z|=1$. Since on $|z|=1$,

$$
\left|\frac{z\left(\Phi_{n-m-1}-\Psi_{n-m-1}\right)}{\Phi_{n-m-1}^{*}+\Psi_{n-m-1}^{*}}\right|=\left|\frac{\Phi_{n-m-1}^{*}-\Psi_{n-m-1}^{*}}{\Phi_{n-m-1}^{*}+\Psi_{n-m-1}^{*}}\right|
$$

and since by $[\underline{8}$, (10.3)],

$$
\operatorname{Re} \frac{\Psi_{n-m-1}^{*}\left(e^{i \varphi}\right)}{\Phi_{n-m-1}^{*}\left(e^{i \varphi}\right)}>0
$$

it follows that the last expression in (50) is less than 1 and thus (49) is proved. Since $q_{m}$ has all zeros in $\mathbb{D}$ there holds $\left|q_{m}(z) / q_{m}^{*}(z)\right|<1$ on $\overline{\mathbb{D}}$, which implies by (49) and (48) that $p_{n-1}^{*}$ has no zero in $\overline{\mathbb{D}}$. Finally, relation (35) follows with the help of the relations (see [22, Prop. 3.2.9])

$$
\Phi_{n-m-1}(z) F(z)+\Psi_{n-m-1}(z)=O\left(z^{n-m-1}\right)
$$

and

$$
\Phi_{n-m-1}^{*}(z) F(z)-\Psi_{n-m-1}^{*}(z)=O\left(z^{n-m}\right)
$$

and (47).

d) $\Rightarrow$ c) The proof runs as in [17, p. 939]. To make the paper self-contained we sketch it. According to $[8$, Theorem 18.2] there exists a function $h: \mathbb{C} \rightarrow \mathbb{C}$ which is analytic in $\mathbb{D}$ and satisfies the inequality $|h(z)|<1$ for $z \in \mathbb{D}$, such that

$$
\frac{\eta p_{n-1}^{*}(z)-z p_{n-1}(z)}{\eta p_{n-1}^{*}(z)+z p_{n-1}(z)}=-\frac{z \Psi_{n-1-m}(z) h(z)-\Psi_{n-m-1}^{*}(z)}{z \Phi_{n-1-m}(z) h(z)+\Phi_{n-m-1}^{*}(z)} .
$$

Isolating $h$ from this equality we find that $h$ can be represented by

$$
h(z)=\frac{q_{l}(z)}{q_{l}^{*}(z)}, \quad \text { where } q_{l}(z)=\prod_{i=1}^{l}\left(z-z_{i}\right), \quad z_{i} \in \mathbb{D},
$$

$q_{l}$ has real coefficients, and $l \geq m$. 
Let us assume that $l>m$. Then it follows from (54) that $-z \Psi_{n-1-m} h+\Psi_{n-m-1}^{*}$ and $z \Phi_{n-1-m} h+\Phi_{n-m-1}^{*}$ have $(l-m)$ common zeros on $|z|=1$, which implies that

$$
\frac{\Phi_{n-m-1}}{\Phi_{n-m-1}^{*}}+\frac{\Psi_{n-m-1}}{\Psi_{n-m-1}^{*}}
$$

has $(l-m)$ zeros on $|z|=1$. But this is impossible, since (see [8, p. 4])

$$
\Omega_{n-m-1} \Psi_{n-m-1}^{*}+\Psi_{n-m-1} \Phi_{n-m-1}^{*}=K z^{n-1-m},
$$

where $K \in \mathbb{R}^{+}$.

a) $\Rightarrow$ d) Let

$$
c_{k}=\frac{1}{2 \pi} \int_{0}^{2 \pi} e^{-i k \varphi} d \sigma(\varphi)=\sum_{s=1}^{n} \mu_{s} e^{-i k \varphi_{s}} \quad \text { for } k=0, \ldots, n-m-1 .
$$

Hence, by expansion at $z=0$,

$$
-\sum_{s=1}^{n} \mu_{s} \frac{z+e^{i \varphi_{s}}}{z-e^{i \varphi_{s}}}=1+2 \sum_{k=1}^{n-1-m} c_{k} z^{k}+O\left(z^{n-m}\right) .
$$

Now the left-hand side is a rational function with real part zero on $|z|=1$ whose numerator and denominator is a polynomial of degree $n$. Thus it may be written as

$$
\frac{\eta p_{n}^{*}(z)-p_{n}(z)}{\eta p_{n}^{*}(z)+p_{n}(z)}=-\sum_{s=1}^{n} \mu_{s} \frac{z+e^{i \varphi_{s}}}{z-e^{i \varphi_{s}}}=1+\ldots,
$$

hence

$$
p_{n}(z)=z p_{n-1}(z) .
$$

Moreover, by (60), partial fraction expansion and the suppositions on $\mu_{s}$, and using $z \frac{d}{d z} f(z)=-i \frac{d}{d \varphi} f\left(e^{i \varphi}\right)$, we obtain that

$$
\mu_{s}=\frac{\left(\eta p_{n}^{*}-p_{n}\right)\left(e^{i \varphi_{s}}\right)}{2 i \frac{d}{d \varphi}\left(p_{n}+\eta p_{n}^{*}\right)\left(e^{i \varphi}\right)_{\varphi=\varphi_{s}}}>0 .
$$

Thus the trigonometric polynomials

$$
\operatorname{Im}\left\{\eta^{-1 / 2} e^{-i \frac{n}{2} \varphi} p_{n}\left(e^{i \varphi}\right)\right\} \text { and } \operatorname{Re}\left\{\eta^{-1 / 2} e^{-i \frac{n}{2} \varphi} p_{n}\left(e^{i \varphi}\right)\right\}
$$

have strictly interlacing zeros, which implies by the argument principle that $p_{n}$ has all zeros in $\mathbb{D}$. By (59) and (60) the implication is proved.

d) $\Rightarrow$ a) Since $p_{n}:=z p_{n-1}$ has all zeros in $\mathbb{D}$ it follows that $\eta p_{n}^{*} \pm p_{n}$ has all zeros in $\partial \mathbb{D}$ and that the zeros of $\eta p_{n}^{*}-p_{n}$ and $\eta p_{n}^{*}+p_{n}$ strictly interlace. Hence, (62) and thus (60) holds with $\mu_{s}>0$ for $s=1, \ldots, n$. Expanding the second expression from (60) in a series at $z=0$ part a) follows.

Obviously, Theorem 3.1 could be proved in a shorter way, that is, by showing the implications $\mathrm{a}) \Rightarrow \mathrm{b}) \Rightarrow \cdots \Rightarrow \mathrm{d}) \Rightarrow \mathrm{a}$ ) only. But by proving all equivalences separately we get additional insights, for instance: 
Remark 3.2. In the proof of Theorem 3.1, c) $\Rightarrow$ b) we have shown that $\tilde{\Phi}_{n-1}$ is generated by the recurrence coefficients $a_{0}, \ldots, a_{n-m-1}, b_{m-1}, \ldots, b_{0}$ when $q_{m}$ is generated by

$$
q_{j}(z)=z q_{j-1}(z)-\eta \bar{b}_{j-1} q_{j-1}^{*}(z) \quad j=1, \ldots, m
$$

$q_{0}(z)=1$, with $\left|b_{j}\right|<1, j=0, \ldots, m-1$. Note that every polynomial $q_{m}$ which has all zeros in $\mathbb{D}$ can be generated by a recurrence relation of the form (6); see [8, Thm. 9.2].

The ideas of proof for Theorem $3.1 \mathrm{k}$ ) and d) go back essentially to the author's papers [16, 17] where positive of on $[-1,1]$ have been characterized by transforming the problem to the unit circle. For the connection with qf on $[-1,1]$ see Section 5 below.

Finally, we point out that recurrence relation (30), under condition (31), offers a simple, constructive way to generate positive trigonometric $(n-1-m, n, d \sigma)$ qf.

With the help of Theorem 3.1 we get easily some information on the location of the nodes of positive qf on the unit circle.

Corollary 3.3. Let $l, m \in \mathbb{N}_{0}, m \leq l$, and let $z \Phi_{n-1-l}+\kappa \Phi_{n-1-l}^{*}=\prod_{\nu=1}^{n-1-l}(z-$ $\left.e^{i \psi_{\nu}}\right), \kappa \in \mathbb{T}$, be a Szegö polynomial. Suppose that $T_{n}(z)=\prod_{s=1}^{n}\left(z-e^{i \varphi_{s}}\right)$ generates a positive $(n-1-m, n, d \sigma) q f$. Then in every interval $\left(\psi_{\nu}, \psi_{\nu+1}\right), \nu=0, \ldots, l$, there is at least one $\varphi_{s}$.

Furthermore, every common zero of $T_{n}$ and $z \Phi_{n-1-l}+\kappa \Phi_{n-1-l}^{*}$ is a zero of $\kappa q_{l}(z)-\eta q_{l}^{*}(z)$, where $q_{l}$ and $\eta$ is given by the representation $T_{n}=z q_{l} \Phi_{n-1-l}+$ $\eta q_{l}^{*} \Phi_{n-1-l}^{*}$.

Proof. By Theorem 3.1 and the obvious fact that every $(n-1-m, n, d \sigma)$ qf is a $(n-1-l, n, d \sigma)$ qf for $l \geq m, T_{n}$ has a representation of the form

$$
T_{n}=z q_{l} \Phi_{n-1-l}+\eta q_{l}^{*} \Phi_{n-1-l}^{*}
$$

where $q_{l}$ has all zeros in $\mathbb{D}$. Hence, $T_{n}\left(e^{i \varphi_{s}}\right)=0$ if and only if for some $k \in \mathbb{Z}$,

(65) $\arg \eta+(2 k+1) \pi=\arg \frac{z q_{l} \Phi_{n-1-l}}{q_{l}^{*} \Phi_{n-1-l}^{*}}\left(e^{i \varphi_{s}}\right)=\arg \frac{q_{l}}{q_{l}^{*}}\left(e^{i \varphi_{s}}\right)+\arg \frac{z \Phi_{n-1-l}}{\Phi_{n-1-l}^{*}}\left(e^{i \varphi_{s}}\right)$

and the condition for a zero of the Szegö polynomial looks similar. Now recall that the argument of a Blaschke product is strictly increasing on $|z|=1$ with respect to $\varphi$. Thus on $\left[\psi_{\nu}, \psi_{\nu+1}\right]$, arg $\frac{z \Phi_{n-1-l}}{\Phi_{n-1-l}^{*}}$ increases by $2 \pi$ and, taking a look at the RHS of (65), $\arg \frac{z q_{l} \Phi_{n-1-l}}{q_{l}^{*} \Phi_{n-1-l}^{*}}$ increases by more than $2 \pi$ on $\left[\psi_{\nu}, \psi_{\nu+1}\right]$, that is, $T_{n}$ has at least one zero in $\left(\psi_{\nu}, \psi_{\nu+1}\right)$ and the assertion is proved.

For the special case that $T_{n}=z \Phi_{n-1}+\eta \Phi_{n-1}^{*}$ the zero property given in the above corollary was proved by Simon [24, Theorem 2.3] by a completely different approach. For related statements in the real case see [2, 18, 19, 25].

For the convenience of the reader let us reformulate parts of Theorem 3.1 in terms of trigonometric polynomials.

Corollary 3.4. $\varphi_{1}, \ldots, \varphi_{n}, 0 \leq \varphi_{1}<\varphi_{2}, \ldots, \varphi_{n}<2 \pi$, are the nodes of a positive trigonometric $(n-1-m, n, d \sigma)$ quadrature formula if and only if there exists a polynomial $\tilde{\Phi}_{n-1}(z)$ satisfying (30) and (31) and $\eta \in \mathbb{T}$ such that, $z=e^{i \varphi}$,

$$
\eta^{-1 / 2} z^{-n / 2} \prod_{s=1}^{n}\left(z-e^{i \varphi_{s}}\right)=2 \operatorname{Re}\left\{z^{-\frac{n}{2}} \eta^{1 / 2} \tilde{\Phi}_{n-1}^{*}(z)\right\} .
$$


Proof. The statement follows immediately by Theorem 3.1 a) and b) and the obvious relations, $z=e^{i \varphi}$,

$$
\begin{aligned}
& \eta^{-1 / 2} z^{-\frac{n}{2}}\left[z \tilde{\Phi}_{n-1}(z)+\eta \tilde{\Phi}_{n-1}^{*}(z)\right]=2 \operatorname{Re}\left\{z^{-\frac{n}{2}+1} \eta^{-1 / 2} \tilde{\Phi}_{n-1}(z)\right\} \\
& =2 \operatorname{Re}\left\{z^{-\frac{n}{2}} \eta^{1 / 2} \tilde{\Phi}_{n-1}^{*}(z)\right\} .
\end{aligned}
$$

We need the following lemma which can be extracted easily from [26] or [9, pp. 144-145].

Lemma 3.5. Let $\mu$ be a positive measure on $[0,2 \pi]$, let $\tilde{n} \in \mathbb{N}, \gamma \in\{0,1 / 2\}$ and $n=2(\tilde{n}+\gamma)$. Let $P_{n-1}(z)=z^{n-1}+\ldots$ be such that

$$
\int_{0}^{2 \pi} e^{-i k \varphi} P_{n-1}\left(e^{i \varphi}\right) d \mu(\varphi)=0 \text { for } k=0, \ldots, n-2 .
$$

Then for any $\eta \in \mathbb{T}$,

$$
\int_{0}^{2 \pi} t(\varphi) \operatorname{Re}\left\{\eta^{1 / 2} e^{-i \frac{n}{2} \varphi} P_{n-1}^{*}\left(e^{i \varphi}\right)\right\} d \mu(\varphi)=0 \text { for } t \in \mathcal{T}_{\tilde{n}-1, \gamma}
$$

Proof. Relation (69) is equivalent to

$$
\begin{aligned}
& \int_{0}^{2 \pi} e^{-i\left(\frac{n}{2}-1 \mp(k+\gamma)\right) \varphi} P_{n-1}\left(e^{i \varphi}\right) d \mu(\varphi) \\
+ & \int_{0}^{2 \pi} e^{-i\left(\frac{n}{2} \mp(k+\gamma)\right) \varphi} P_{n-1}^{*}\left(e^{i \varphi}\right) d \mu(\varphi)=0 \text { for } k=0, \ldots, \tilde{n}-1 .
\end{aligned}
$$

Now the first integral is zero by (68) and thus the second integral also by taking the complex conjugate of it.

With the help of the above lemma we get an explicit weight function depending on $n$ with respect to which the trigonometric nodes polynomial has maximal orthogonality. Furthermore, we obtain the orthogonality property (12) of the nodes polynomial (66) by an approach via OPUC's.

Corollary 3.6. Let $n=2(\tilde{n}+\gamma)$, where $\tilde{n} \in \mathbb{N}$ and $\gamma \in\{0,1 / 2\}$. If $\varphi_{1}, \ldots, \varphi_{n}$, $0 \leq \varphi_{1}<\varphi_{2}, \ldots, \varphi_{n}<2 \pi$, are the nodes of a positive trigonometric $(n-1-m, n, d \sigma)$ $q f$, then there exists a polynomial $q_{m}(z)$ which has all zeros in $|z|<1$ and $\eta \in \mathbb{T}$ such that $\operatorname{Re}\left\{\eta^{1 / 2} z^{-\frac{n}{2}}\left(q_{m}^{*} \Phi_{n-1-m}^{*}\right)(z)\right\}, z=e^{i \varphi}$, is the nodes polynomial and

$$
\begin{aligned}
& \int_{0}^{2 \pi} t(\varphi) \operatorname{Re}\left\{\eta^{1 / 2} e^{-i \frac{n}{2} \varphi}\left(q_{m}^{*} \Phi_{n-1-m}^{*}\right)\left(e^{i \varphi}\right)\right\} \frac{d \varphi}{\left|\left(q_{m}^{*} \Phi_{n-1-m}^{*}\right)\left(e^{i \varphi}\right)\right|^{2}} \\
& =0 \text { for } t \in \mathcal{T}_{\tilde{n}-1, \gamma} .
\end{aligned}
$$

Moreover,

$$
\int_{0}^{2 \pi} t(\varphi) \operatorname{Re}\left\{\eta^{1 / 2} e^{-i \frac{n}{2} \varphi}\left(q_{m}^{*} \Phi_{n-1-m}^{*}\right)\left(e^{i \varphi}\right)\right\} d \sigma(\varphi)=0 \text { for } t \in \mathcal{T}_{\tilde{n}-1-m, \gamma} .
$$

Proof. By Theorem $3.1 q_{m} \Phi_{n-1-m}$ has all zeros in $\mathbb{D}$; hence, see e.g. [8, 25],

$$
\int_{0}^{2 \pi} e^{-i k \varphi}\left(q_{m} \Phi_{n-1-m}\right)\left(e^{i \varphi}\right) \frac{d \varphi}{\left|\left(q_{m}^{*} \Phi_{n-1-m}^{*}\right)\left(e^{i \varphi}\right)\right|^{2}}=0 \text { for } k=0, \ldots, n-2 .
$$

Applying Lemma 3.5 the orthogonality property (71) follows. 
Since $\left|q_{m}^{*}\left(e^{i \varphi}\right)\right|^{2}$ is from $\mathcal{T}_{m}$ we obtain from (71), by putting $t(\varphi)=\tilde{t}(\varphi)\left|q_{m}^{*}\left(e^{i \varphi}\right)\right|^{2}$, $\tilde{t} \in \mathcal{T}_{\tilde{n}-1-m, \gamma}$, that (72) holds with respect to the measure $d \varphi /\left|\Phi_{n-1-m}^{*}\left(e^{i \varphi}\right)\right|^{2}$ instead of $d \sigma$; but, see [7, Thm 2.2, p. 198],

$$
\int_{0}^{2 \pi} e^{ \pm i k \varphi} d \sigma(\varphi)=\int_{0}^{2 \pi} e^{ \pm i k \varphi} \frac{d \varphi}{\left|\Phi_{n-1-m}^{*}\left(e^{i \varphi}\right)\right|^{2}} \text { for } k=0, \ldots, n-1-m
$$

and thus relation (72) follows, taking into consideration the fact that the orthogonality property (72) depends on the first $n-m-1=2 \tilde{n}+2 \gamma-m-1$ trigonometric moments of $d \sigma$ only.

We note that in general $\operatorname{Im}\left\{z^{-\frac{n}{2}} \eta^{1 / 2} \tilde{\Phi}_{n-1}^{*}(z)\right\}, \tilde{\Phi}_{n-1}$ given by (30) and (31), does not coincide with $\operatorname{Im}\left\{z^{-\frac{n}{2}} \eta^{1 / 2}\left(q_{m}^{*} \Phi_{n-1-m}^{*}\right)(z)\right\}$, since

$$
z \tilde{\Phi}_{n-1}-\eta \tilde{\Phi}_{n-1}^{*}=z \tilde{q}_{m} \Phi_{n-1-m}-\eta \tilde{q}_{m}^{*} \Phi_{n-1-m}^{*},
$$

where $\tilde{q}_{m}$ is given by $\tilde{q}_{j}(z)=z \tilde{q}_{j-1}(z)+\eta \overline{\tilde{a}}_{n-1-j} \tilde{q}_{j-1}^{*}(z), j=1,2, \ldots, m$ with $\tilde{q}_{0}(z)=$ 1 , which should be compared with (43).

\section{Asymptotics of weights}

As a consequence of Theorem 3.1 we obtain the following representation of the quadrature weights needed in what follows. Note that only the $\Phi_{n}$ 's and $q_{m}$ 's and no $\Psi_{n}$ 's appear in the representation.

Corollary 4.1. The quadrature weights $\mu_{s}$ from (29) can be represented in the form

$$
\mu_{s}=\frac{-\eta K_{n-m-1} z_{s}^{n-1}\left|q_{m}\left(z_{s}\right)\right|^{2}}{\left(z \Phi_{n-m-1} q_{m}-\eta \Phi_{n-m-1}^{*} q_{m}^{*}\right)\left(z_{s}\right)\left(z \Phi_{n-m-1} q_{m}+\eta \Phi_{n-m-1}^{*} q_{m}^{*}\right)^{\prime}\left(z_{s}\right)}
$$

where $z_{s}=e^{i \varphi_{s}}$ and $K_{n-m-1}=2 \prod_{j=0}^{n-m-2}\left(1-\left|a_{j}\right|^{2}\right)$.

Proof. By (9), (44) and (45) it follows that

$$
\mu_{s}=\frac{1}{2 z_{s}} \frac{\left(z \Psi_{n-m-1} q_{m}-\eta \Psi_{n-m-1}^{*} q_{m}^{*}\right)\left(z_{s}\right)}{\left(z \Phi_{n-m-1} q_{m}+\eta \Phi_{n-m-1}^{*} q_{m}^{*}\right)^{\prime}\left(z_{s}\right)} .
$$

Now, at the zeros of $z \Phi_{n-m-1} q_{m}+\eta \Phi_{n-m-1}^{*} q_{m}^{*}$ we have

$$
\begin{gathered}
\left(z \Phi_{n-m-1} q_{m}-\eta \Phi_{n-m-1}^{*} q_{m}^{*}\right)\left(z \Psi_{n-m-1} q_{m}-\eta \Psi_{n-m-1}^{*} q_{m}^{*}\right) \\
=-2 \eta z q_{m} q_{m}^{*}\left(\Phi_{n-m-1} \Psi_{n-m-1}^{*}+\Psi_{n-m-1} \Phi_{n-m-1}^{*}\right) \\
=-2 \eta z q_{m} q_{m}^{*} K_{n-m-1} z^{n-m-1}
\end{gathered}
$$

where in the last equality we used the known fact that [8, (5.6)]

$$
\Phi_{n-m-1} \Psi_{n-m-1}^{*}+\Psi_{n-m-1} \Phi_{n-m-1}^{*}=K_{n-m-1} z^{n-m-1}
$$

which gives by (74) the assertion.

Theorem 4.2. Let $d \sigma(\varphi)=f(\varphi) d \varphi$ be positive and from $\operatorname{Lip} \gamma, 0<\gamma \leq 1$ on $[\alpha, \beta] \subseteq[0,2 \pi]$. Suppose that $\varphi_{1, n}, \ldots, \varphi_{n, n}, 0 \leq \varphi_{1, n}<\varphi_{2, n}<\cdots<\varphi_{n, n}<2 \pi$ generates a sequence of positive $(n-m(n)-1, n, w)$ qf with quadrature weights $\mu_{s, n}$. Furthermore, let us assume that the associated $q_{m(n)}$ satisfy uniformly on $[\alpha, \beta]$,

$$
\lim _{n}\left(1-\frac{2}{n} \operatorname{Re}\left\{e^{i \varphi} \frac{q_{m(n)}^{*^{\prime}}\left(e^{i \varphi}\right)}{q_{m(n)}^{*}\left(e^{i \varphi}\right)}\right\}\right)=g(\varphi) .
$$


Then uniformly for $\varphi_{s, n} \in[\alpha+\varepsilon, \beta-\varepsilon], \varepsilon>0$,

$$
\frac{1}{n \mu_{s, n}}=\frac{g\left(\varphi_{s, n}\right)}{f\left(\varphi_{s, n}\right)}+o(1) .
$$

Proof. First let us note that (73) can be written in the form

$$
\begin{aligned}
& -4 \mu_{s}= \\
& \frac{K_{n-m-1}\left|q_{m}\left(e^{i \varphi_{s}}\right)\right|^{2}}{\operatorname{Im}\left\{\overline{e^{-i \frac{n}{2} \varphi_{s}}\left(\eta^{1 / 2} q_{m}^{*} \Phi_{n-m-1}^{*}\right)\left(e^{i \varphi_{s}}\right)}\right\} \frac{d}{d \varphi} \operatorname{Re}\left\{e^{-i \frac{n}{2} \varphi}\left(\eta^{1 / 2} q_{m}^{*} \Phi_{n-m-1}^{*}\right)\left(e^{i \varphi}\right)\right\}_{\varphi=\varphi_{s}}} .
\end{aligned}
$$

Now

$$
\begin{aligned}
& \operatorname{Im}\left\{\overline{e^{-i \frac{n}{2} \varphi}\left(\eta^{1 / 2} q_{m}^{*} \Phi_{n-m-1}^{*}\right)\left(e^{i \varphi}\right)}\right\} \frac{d}{d \varphi} \operatorname{Re}\left\{e^{-i \frac{n}{2} \varphi}\left(\eta^{1 / 2} q_{m}^{*} \Phi_{n-m-1}^{*}\right)\left(e^{i \varphi}\right)\right\} \\
- & \operatorname{Re}\left\{e^{-i \frac{n}{2} \varphi}\left(\eta^{1 / 2} q_{m}^{*} \Phi_{n-m-1}^{*}\right)\left(e^{i \varphi}\right)\right\} \frac{d}{d \varphi} \operatorname{Im}\left\{e^{-i \frac{n}{2} \varphi}\left(\eta^{1 / 2} q_{m}^{*} \Phi_{n-m-1}^{*}\right)\left(e^{i \varphi}\right)\right\} \\
= & \left.\operatorname{Im}\left\{\overline{\left(e^{-i \frac{n}{2} \varphi}\left(\eta^{1 / 2} q_{m}^{*} \Phi_{n-m-1}^{*}\right)\left(e^{i \varphi}\right)\right.}\right) \frac{d}{d \varphi}\left(e^{-i \frac{n}{2} \varphi}\left(\eta^{1 / 2} q_{m}^{*} \Phi_{n-m-1}^{*}\right)\left(e^{i \varphi}\right)\right)\right\} \\
= & \operatorname{Im}\left\{-i \frac{n}{2}\left|\left(q_{m}^{*} \Phi_{n-m-1}^{*}\right)\left(e^{i \varphi}\right)\right|^{2}+\overline{\left(q_{m}^{*} \Phi_{n-m-1}^{*}\right)\left(e^{i \varphi}\right)} \frac{d}{d \varphi}\left(\left(q_{m}^{*} \Phi_{n-m-1}^{*}\right)\left(e^{i \varphi}\right)\right)\right\}
\end{aligned}
$$

and thus, since the $e^{i \varphi_{s}}$ are the zeros of $\operatorname{Re}\left\{e^{-i \frac{n}{2} \varphi}\left(\eta^{1 / 2} q_{m}^{*} \Phi_{n-m-1}^{*}\right)\left(e^{i \varphi}\right)\right\}$, we may replace the denominator in (77) by the last expression from (78) which yields

$$
\mu_{s}\left|\left(q_{m}^{*} \Phi_{n-m-1}^{*}\right)\left(e^{i \varphi_{s}}\right)\right|^{2}=\frac{K_{n-m-1}\left|q_{m}^{*}\left(e^{i \varphi_{s}}\right)\right|^{2}}{4 \operatorname{Im}\left\{i \frac{n}{2}-i e^{i \varphi_{s}\left(q_{m}^{*} \Phi_{n-m-1}^{*}\right)^{\prime}\left(e^{i \varphi_{s}}\right)}\right.} .
$$

Now by the assumptions on $f$ it is known (see e.g. 25, 22 ) that uniformly on $[\alpha+\varepsilon, \beta-\varepsilon], \varepsilon>0$,

$$
\lim _{n \rightarrow \infty} \frac{K_{n-m(n)-1}}{2\left|\Phi_{n-m(n)-1}^{*}\left(e^{i \varphi}\right)\right|^{2}}=\frac{1}{\left|D\left(e^{i \varphi}, \sigma\right)\right|^{2}}=f(\varphi)
$$

taking into consideration that

$$
\lim _{n} K_{n-m(n)-1} / 2=1 / D(0, \sigma),
$$

where $D(z, \sigma)$ is the so-called Szegö function, that is,

$$
D(z)=\exp \left\{\frac{1}{2 \pi} \int_{0}^{2 \pi} \frac{e^{i \varphi}+z}{e^{i \varphi}-z} \log f(\varphi) d \varphi\right\} .
$$

Next let us prove that (80) and (81) imply that uniformly on $[\alpha+\varepsilon, \beta-\varepsilon], \varepsilon>0$,

$$
\frac{1}{n}\left|\frac{\Phi_{2 n-1-m}^{*^{\prime}}\left(e^{i \varphi}\right)}{\Phi_{2 n-1-m}^{*}\left(e^{i \varphi}\right)}\right| \underset{n \rightarrow \infty}{\longrightarrow} 0 .
$$


Indeed, by the local version of Bernstein's inequality

$$
\begin{aligned}
\max _{\varphi \in[\alpha+\varepsilon, \beta-\varepsilon]} & \left|\frac{d}{d \varphi} \Phi_{2 n-m-1}^{*}\left(e^{i \varphi}\right)\right| \leq \text { const } \\
& \times\left((2 n-m-1) \max _{\varphi \in[\alpha, \beta]}\left|\Phi_{2 n-m-1}^{*}\left(e^{i \varphi}\right)-\Phi_{[\sqrt{2 n-m-1}]}^{*}\left(e^{i \varphi}\right)\right|\right. \\
& \left.+\sqrt{2 n-m-1} \max _{\varphi \in[\alpha, \beta]}\left|\Phi_{[\sqrt{2 n-m-1}]}^{*}\left(e^{i \varphi}\right)\right|\right)
\end{aligned}
$$

which gives by (80) and (81), in conjunction with the facts that $D\left(e^{i \varphi}\right) \neq 0$ on $[\alpha, \beta]$ and $m(n) \leq n$, relation (83).

\section{Connection to positive QF on $[-1,1]$}

Remark 5.1. Let $m, n \in \mathbb{N}_{0}, 0 \leq m \leq 2 n-1$. Obviously, using the symmetry with respect to $\pi$ we have

$$
\int_{-1}^{+1} p(x) d \psi(x)=\sum_{s=1}^{n} \lambda_{s} p\left(x_{s}\right) \quad \text { for } p \in \mathbb{P}_{2 n-1-m},
$$

where $-1<x_{n}<\cdots<x_{1}<1$ and $\lambda_{1}, \ldots, \lambda_{n} \in \mathbb{R}^{+}$, if and only if

$$
\int_{-\pi}^{+\pi} t(\varphi) d \sigma(\varphi)=\sum_{s=1}^{n} \lambda_{s}\left(t\left(\varphi_{s}\right)+t\left(-\varphi_{s}\right)\right) \quad \text { for } t \in \mathcal{T}_{2 n-1-m}
$$

where the $\varphi_{s}=\arccos x_{s}$ satisfy $0<\varphi_{1}<\varphi_{2}<\cdots<\varphi_{n}<\pi$, and $\sigma$ is given by

$$
\sigma(\varphi)= \begin{cases}\psi(1)-\psi(\cos \varphi) & \text { for } 0 \leq \varphi \leq \pi \\ \psi(\cos \varphi)-\psi(1) & \text { for }-\pi \leq \varphi \leq 0\end{cases}
$$

Note that, $x=\frac{1}{2}\left(z+\frac{1}{z}\right)$,

$$
2^{n} z^{n} \prod_{s=1}^{n}\left(x-x_{s}\right)=\prod_{s=1}^{n}\left(z-e^{i \varphi_{s}}\right)\left(z-e^{-i \varphi_{s}}\right) .
$$

By this equivalence and Theorem 3.1 we obtain immediately a complete description of positive of of degree of exactness $2 n-1-m$ on $[-1,1]$. Indeed, Theorem 3.1k) and d) will lead to characterizations given by the author in [16. In fact, in [16] we transformed the problem in the proof to the unit circle and solved it there, that is, we proved Theorem 3.1 ) and d) in the real case and transformed it back. It can be shown that the characterization by the recurrence relation, that is, by Theorem 3.1b) yields, using the connection between the recurrence coefficients of OPUC's and the recurrence coefficients of polynomials orthogonal on $[-1,1]$ (see [23. (13.1.7)] or [8, Thm. 31.1]), the characterization of positive qf on $[-1,1]$ by the three-term recurrence relation given in [17, 19] by the author; see also [20].

When we consider Radau or Lobatto qf on $[-1,1]$ (see [20, Section 4]) that is, that one or two nodes are the boundary points \pm 1 the corresponding equivalence is as follows: Let us consider the Radau case, that is, the boundary point 1 is a node - analogously the other cases are obtained - then

$$
\int p(x) d \psi(x)=\sum_{s=1}^{n} \lambda_{s} p\left(x_{s}\right)+\lambda_{0} p(+1) \quad \text { for } p \in \mathbb{P}_{2 n-m},
$$


where $-1<x_{n}<\cdots<x_{1}<1$ and $\lambda_{0}, \lambda_{1}, \ldots, \lambda_{n} \in \mathbb{R}^{+}$, if and only if

$$
\int_{-\pi}^{+\pi} t(\varphi) d \sigma(\varphi)=\frac{\lambda_{0}}{2} t\left(\varphi_{0}\right)+\sum_{s=1}^{n} \lambda_{s}\left(t\left(\varphi_{s}\right)+t\left(-\varphi_{s}\right)\right) \quad \text { for } t \in \mathcal{T}_{2 n-m},
$$

where the $\varphi_{s}=\arccos x_{s}$ satisfy $\varphi_{0}=0<\varphi_{1}<\varphi_{2}<\cdots<\varphi_{n}<\pi$ and where $\lambda_{0}, \lambda_{1}, \ldots, \lambda_{n} \in \mathbb{R}^{+}$.

\section{REFERENCES}

[1] N.I. Achieser and M. Krein, The L-problem of moments, In: Achieser, N.I., Krein, M. (eds.) Some Questions in the Theory of Moments. Translation of Mathematical Monographs, Vol. 2, Amer. Math. Soc., Providence, RI, 1962. MR0167806 (29:5073)

[2] A.F. Beardon and K.A. Driver, The zeros of linear combinations of orthogonal polynomials, J. Approx. Theory 137 (2005), no.2, 179-186. MR2186945 (2006m:26025)

[3] R. Cruz-Barroso, P. González Vera and O. Njåstad, On bi-orthogonal systems of trigonometric functions and quadrature formulas for periodic integrals, Numer. Alg. 44 (2007), 309-333. MR2335805 (2008i:42003)

[4] C.F. Bracciali, Xin Li and A. Sri Ranga, Real orthogonal polynomials in frequency analysis, Math. Comp. 74(249), 341-362 (2004). MR2085896 (2005e:94014)

[5] M.J. Cantero, L. Moral and L. Velázquez, Measures and para-orthogonal polynomials on the unit circle, East J. Approx. 8 (2002), 447-464. MR.1952510 (2004i:42021)

[6] L. Daruis, P. González-Vera and O. Njåstad, Szegő quadrature formulas for certain Jacobi type weight functions, Math. Comp. 71, 683-701 (2002). MR.1885621 (2002k:41043)

[7] G. Freud, Orthogonal Polynomials, Pergamon Press, Oxford (1971).

[8] Ya.L. Geronimus, Polynomials orthogonal on a circle and their applications, Amer. Math. Soc. Transl. 3, 1-78 (1962). MR0061706 (15:869i)

[9] Ya.L. Geronimus, Orthogonal Polynomials, Consultants Bureau, New York, 1961. MR0133643 (24:A3469)

[10] L. Golinskii, Quadrature formula and zeros of para-orthogonal polynomials on the unit circle, Acta Math. Hungar. 96(3), 169-186 (2002). MR.1919160 (2003e:41048)

[11] W.B. Gragg, Positive definite Toeplitz matrices, the Arnoldi process for isometric operators, and Gaussian quadrature on the unit circle (in Russian), In: Nicholaev, E.S. (ed.) Numerical Methods in Linear Algebra, pp. 16-32. Moscow University Press, Moscow (1982). Published in English in slightly modified form in J. Comput. Appl. Math. 46, 183-198 (1993). MR1222480 (94e:65046)

[12] D. Jackson, Series of orthogonal polynomials and orthogonal trigonometric sums, Annals of Mathematics (2) 34 (1933), 527-545, 799-814. MR.1503122

[13] W.B. Jones, O. Njåstad and W.J. Thron, Moment theory, orthogonal polynomials, quadrature and continued fractions associated with the unit circle, Bull. London Math. Soc. 21, 113-152 (1989). MR 976057(90e:42027)

[14] Sun-Mi Kim and L. Reichel, Anti-Szegő quadrature rules, Math. Comp. 76(258), 795-810 (2007). MR 2291837 (2008e:65085)

[15] G.V. Milovanović, A.S. Cvetković and M.P. Stanić, Trigonometric orthogonal systems and quadrature formulae, Comput. Math. Appl. 56 (2008), no. 11, 2915-2931. MR2467680 (2009i:65038)

[16] F. Peherstorfer, Characterization of positive quadrature formulas, SIAM J. Math. Anal. 12, 935-942 (1981). MR635246 (82m:65021)

[17] F. Peherstorfer, Characterization of quadrature formulas II, SIAM J. Math. Anal. 15, 10211030 (1984). MR755862 (86a:65025)

[18] F. Peherstorfer, Linear combinations of orthogonal polynomials generating positive quadrature formulas, Math. Comp. 55(1990), 231-241. MR.1023052 (90j:65043)

[19] F. Peherstorfer, On positive quadrature formulas, ISNM, 112, Birkäuser, Basel, 297-313 (1993). MR $1248412(94 \mathrm{k}: 65035)$

[20] F. Peherstorfer, Positive quadrature formulas III: Asymptotics of weights, Math. Comp. 77 (2008), 2241-2259. MR2429883 (2009k:65043)

[21] F. Peherstorfer, Szegö-Kronrod quadrature on the unit circle, manuscript. 
[22] B. Simon, Orthogonal polynomials on the unit circle, Part 1: Classical theory, AMS Colloquium Series, American mathematical Society, Providence, RI, 2005. MR2105088 (2006a:42002a)

[23] B. Simon, Orthogonal polynomials on the unit circle, Part 2: Spectral theory, AMS Colloquium Series, American mathematical Society, Providence, RI, 2005. MR 2105089 (2006a:42002b)

[24] B. Simon, Rank one perturbations and the zeros of paraorthogonal polynomials on the unit circle, J. Math. Anal. Appl. 329 (2007), 376-382. MR2306808 (2008c:42027)

[25] G. Szegö, Orthogonal Polynomials, 4th ed., Amer. Math. Soc., Providence, 1975.

[26] G. Szegö, On bi-orthogonal systems of trigonometric polynomials, Magyar Tud. Akad. Mat. Kutat 8(1964), 255-273. MR0166541 (29:3815)

[27] M.-W.L. Wong, First and second kind paraorthogonal polynomials and their zeros, J. Approx. Theory 146 (2007), 282 - 293. MR2328186 (2008f:42028)

Group for Dynamical Systems and Approximation Theory, Institute for Analysis, Johannes Kepler University, A-4040 Linz, Austria 